wording was used. Refusals were highest for 15-19 year olds, families in lower income neighbourhoods, and motor vehicle passenger injuries. After covariate adjustment, passive wording increased likelihood of consent twofold.

Conclusion Caution is needed when interpreting results from studies using hospital samples, especially when wording of consent for follow-up requires direct affirmation.

\title{
0487 BIAS IN HOSPITAL SAMPLES USED FOR INJURY RESEARCH BASED ON CONSENT WORDING
}

B Pless*, B Hagel, G Keays, X Zhang, H Magdalinos Correspondence: Montreal Children's Hospital, McGill University, 2300 Tupper Montreal, OC H3H1P3, Canada

10.1136/ip.2010.029215.487

Background Many injury studies involve samples of hospitalised or emergency room emergency department patients. In studies involving follow-up, consent is required when patients are first identified. When the proportion who refuse is high, the study sample may be biased in ways that distort the validity of the results. This study aimed to determine if wording requiring active consent resulted in more refusals than wording that was essentially passive that is where consent is implied in the absence of direct refusal.

Methods The Canadian Hospital Injury Reporting and Prevention Program (CHIRPP) is based in 14 hospitals, 10 being paediatric. It uses a two-page questionnaire to describe how the injury occurred and what was injured. We chose 5 of the 10 paediatric hospitals; 3 used passive wording and 2 used active wording for consent to follow-up. We employed logistic regression to analyse the effect of wording on consent rates and whether the effect was influenced by patient demographic and clinical characteristics.

Results $63 \%$ of parents gave consent for follow-up at hospitals using passive consent wording versus $53 \%$ where active 